\title{
Assessment of nutritional status in children and adolescents with post-infectious bronchiolitis obliterans
}

\author{
Avaliação do estado nutricional de crianças e adolescentes com bronquiolite \\ obliterante pós-infecciosa

\section{Vera Lúcia Bosa1, Elza Daniel de Mello², Helena Teresinha Mocelin ${ }^{3}$,} \\ Franceliane Jobim Benedetti ${ }^{1}$, Gilberto Bueno Fischer $^{4}$
}

\section{Resumo}

Objetivos: Avaliar o estado nutricional de crianças e adolescentes portadores de bronquiolite obliterante e analisar a associação com aspectos clínicos e nutricionais.

Métodos: Estudo com 57 crianças e adolescentes. Realizou-se avaliação do estado nutricional (nas crianças, pelos escores z de peso/ idade, estatura/idade e peso/estatura; nos adolescentes, por estatura/idade e percentis do índice de massa corporal), da composição corporal (avaliaram-se dobras cutâneas tricipital, subescapular e soma das duas e circunferência muscular do braço) e avaliação da função pulmonar nos maiores de 8 anos.

Resultados: Destaca-se o alto percentual de desnutrição $(21,7 \%)$ e risco de desnutrição $(17,5 \%)$. Nas crianças, o peso/idade e estatura/idade apresentaram maior percentual de desnutrição, 21,6 e $16,2 \%$, respectivamente, ao passo que o peso/estatura subestimou este diagnóstico. Nos adolescentes, o índice de massa corporal demonstrou alto percentual de desnutrição (25\%) e riscode desnutrição (20\%). Na composição corporal, 51\% apresentaram baixa reserva muscular, e a maioria dos pacientes apresentou reserva de gordura dentro da normalidade. O prejuízo da função pulmonar associou-se com menor desempenho ao exercício $(r=0,434 ; p=$ $0,024)$. A desnutrição e/ou risco nutricional e baixa reserva muscular associaram-se significativamente com teste de caminhada de 6 minutos $(p=0,032 ; p=0,030)$. Não houve associação entre a espirometria e variáveis nutricionais $(p>0,05)$.

Conclusão: Estes resultados salientam a necessidade de intervenção nutricional. Na avaliação nutricional, além da utilização dos indicadores de peso e estatura, faz-se necessária a associação da análise da composição corporal, para que um número maior de pacientes com desnutrição e/ou com risco aumentado de desenvolvê-la sejam identificados e adequadamente manejados.

J Pediatr (Rio J). 2008;84(4):323-330: Bronquiolite obliterante, estado nutricional, composição corporal, antropometria, desnutrição, espirometria.

\begin{abstract}
Objective: To assess the nutritional status of children and adolescents with bronchiolitis obliterans and to analyze associations with clinical and nutritional factors.
\end{abstract}

Methods: The study included 57 patients. Nutritional status was assessed using z scores for weight/age, stature/age, weight/stature in children, and stature/age and body mass index percentiles in adolescents. Body composition was assessed via tricipital skin folds, subscapular skin folds, and the sum of both plus the muscular circumference of the arm; pulmonary function was also investigated in subjects over 8 years old.

Results: The high percentages of malnutrition and risk for malnutrition are noteworthy: 21.7 and $17.5 \%$, respectively. Among children, weight/age and stature/age detected higher percentages of malnutrition (21.6 and $16.2 \%$ ), while weight/stature underestimated this diagnosis. Among adolescents, body mass index detected a high percentage of malnutrition (25\%) and of risk for malnutrition $(20 \%)$. Body composition analysis detected $51 \%$ of patients with low muscle reserves, and the majority of patients had normal fat reserves. Compromised pulmonary function was associated with poor performance at exercise $(r=0.434 ; p=0.024)$. Malnutrition and/or nutritional risk and low muscle reserves were significantly associated with the 6 -minute walk test $(p=0.032 ; p=$ 0.030 ). There was no association between spirometry and the nutritional variables $(p>0.05)$.

Conclusions: These results emphasize the need for nutritional intervention, and suggest that, in addition to using weight and height indices for nutritional assessment, it is necessary to combine these with an analysis of body composition, so that a larger number of patients with malnutrition and/or at an increased risk of developing malnutrition may be identified and correctly managed.

J Pediatr (Rio J). 2008;84(4):323-330: Bronchiolitis obliterans, nutritional status, body composition, anthropometry, malnutrition, spirometry.

1. Nutricionista. Mestranda, Programa de Pós-Graduação em Ciências Médicas/Pediatria, Universidade Federal do Rio Grande do Sul (UFRGS), Porto Alegre, RS.

2. Doutora em Pediatria, UFRGS, Porto Alegre, RS. Médica, Serviço de Nutrologia, Hospital de Clínicas de Porto Alegre (HCPA), Porto Alegre, RS.

3. Doutora em Pneumologia, UFRGS, Porto Alegre, RS. Médica, Serviço de Pneumologia Pediátrica, Hospital da Criança Santo Antônio - Irmandade Santa Casa de Misericórdia de Porto Alegre (HCSA-ISCMPA), Porto Alegre, RS.

4. Doutor em Pneumologia, UFRGS, Porto Alegre, RS. Professor titular, Pediatria, Universidade Federal de Ciências da Saúde de Porto Alegre (UFCSPA), Porto Alegre, RS. Médico, Serviço de Pneumologia Pediátrica, HCSA-ISCMPA, Porto Alegre, RS.

Não foram declarados conflitos de interesse associados à publicação deste artigo.

Como citar este artigo: Bosa VL, de Mello ED, Mocelin HT, Benedetti FJ, Fischer GB. Assessment of nutritional status in children and adolescents with post-infectious bronchiolitis obliterans. J Pediatr (Rio J). 2008;84(4):323-330.

Artigo submetido em 26.03.08, aceito em 28.05.08.

doi:10.2223/JPED.1812 


\section{Introdução}

A bronquiolite obliterante pós-infecciosa (BO) é uma conseqüência de agressão ao epitélio do trato respiratório inferior que se caracteriza por obstrução da via aérea distal ${ }^{1}$. A prevalência da BO é desconhecida, mas estudos recentes apontam para um aumento da sua incidência ${ }^{2}$. Em crianças, ocorre com mais freqüência no primeiro ano de vida, estando associada, na maioria das vezes, à bronquiolite viral aguda $(B V A)^{3}$.

Publicações ressaltam que, além do diagnóstico da $\mathrm{BO}$, do tratamento agressivo das infecções e da oxigenoterapia, é importante desenvolver um plano nutricional adequado para evolução clínica favorável desta enfermidade. Apesar de escassa literatura que evidencie a importância especifica de cuidados nutricionais em $\mathrm{BO}$, assim como em outras pneumopatias crônicas nas quais o consumo energético é acentuado, deve-se manter o paciente com aporte energético adequado. Embora essas necessidades devam ser individualizadas, o objetivo é manter o crescimento adequado para a idade do indivíduo ${ }^{2,4}$.

Os poucos estudos disponíveis na literatura ${ }^{4-8}$ que referem a condição nutricional de pacientes com BO são inconsistentes quanto aos métodos utilizados e não exploram a condição nutricional destes indivíduos, mas são unânimes quanto à atenção dispensada na recuperação e/ou preservação do estado nutricional nesta condição clínica e ao reconhecer as implicações que o déficit nutricional pode trazer na evolução da doença.

O objetivo do presente estudo é avaliar o estado nutricional de crianças e adolescentes portadores de BO e analisar a associação com aspectos clínicos e nutricionais.

\section{Métodos}

Realizou-se um estudo transversal no qual a população foi constituída por todas as crianças e adolescentes com diagnóstico de BO em acompanhamento regular nos ambulatórios do Hospital da Criança Santo Antônio e Hospital Materno Infantil Presidente Vargas de Porto Alegre (RS), no período de outubro de 2005 a maio de 2006. O diagnóstico de BO baseou-se na coexistência de: (1) obstrução das vias aéreas persistente após episódio de doença obstrutiva aguda das vias aéreas em uma criança previamente saudável no primeiro ano de vida; (2) imagem tomográfica sugestiva, nomeadamente padrão em mosaico e/ou bronquiectasias; e (3) exclusão de outras doenças pulmonares obstrutivas, como fibrose cística (FC), tuberculose, malformações congênitas e infecção por vírus da imunodeficiência humana ${ }^{8,9}$. Foram incluídos crianças e adolescentes na faixa etária de 1 a 18 anos de idade, de ambos os sexos e que apresentavam condições físicas para realização das medidas antropométricas. Os pacientes foram divididos em crianças e adolescentes, de acordo com a faixa etária ${ }^{10}$.

As medidas antropométricas de peso e estatura foram aferidas utilizando técnicas padronizadas e com equipamentos calibrados $^{11}$.
Nas crianças, o estado nutricional foi determinado pelo escores $z$ de peso por idade (ZPI), estatura por idade (ZEI) e peso por estatura (ZPE). Desnutrição e/ou baixa estatura foi definida por ZPE e ZEI $\leq-2 Z$, e obesidade pelo $Z P E \geq+2 Z^{10}$; risco para desnutrição pelo $Z P E \leq-1,28 Z^{12}$, e o percentil 10 para peso por idade $(P I)^{13}$; e sobrepeso por $Z P E \geq+1,28 Z$. As crianças com ZPE entre $-1,27$ e $+1,27 Z$ foram consideradas eutróficas ${ }^{12}$. Quando foi aplicado mais de um dos índices antropométricos (ZPE, ZPI e ZEI) para a classificação do estado nutricional, em caso de discrepância entre as faixas utilizadas, considerou-se o menor valor.

Entre os adolescentes, analisou-se o ZEI e a distribuição dos percentis do índice de massa corporal (IMC). Para classificar desnutrição e/ou baixa estatura, definiu-se ZEI $<-2 Z$. $\mathrm{Na}$ distribuição em percentis do IMC, seguiu-se a classificação da Organização Mundial da Saúde $(\mathrm{OMS})^{10}$. Como esta não classifica risco nutricional para desnutrição, adotou-se o proposto por Frisancho ${ }^{11}$. Aplicou-se a auto-avaliação do estágio de maturação sexual ${ }^{14}$, e os adolescentes foram classificados em pré-púberes, púberes ou pós-púberes ${ }^{10}$.

Entre os $\leq 5$ anos, os dados foram analisados com auxílio do programa Anthro da OMS, versão beta de 17/02/06 ${ }^{15}$; a partir dos 5 anos, com auxílio do programa Epi-Info, versão 3.3.2 de 09/02/05 com base na referência do Centers for Disease Control $(C D C)^{16}$. Para os percentis de IMC, também foram realizadas comparações com outros valores de referência ${ }^{17,18}$.

Na avaliação da composição corporal, foram analisadas as dobras cutâneas tricipital (DCT), subescapular (DCS) e a soma das duas (SDCTS) para reserva de gordura, além da circunferência muscular do braço (CMB) para determinar reserva muscular ${ }^{11}$. A medida da circunferência braquial $(C B)$ foi realizada com fita métrica inextensível da marca Secca ${ }^{\circledR}$ e, para a mensuração das dobras cutâneas, foi utilizado compasso científico Lange ${ }^{\circledR}$.

A função pulmonar foi avaliada em pacientes maiores de 8 anos, realizando a espirometria e o teste de caminhada de 6 minutos (TC6). A espirometria foi realizada com um espirômetro portátil (SpiroDoc v.3.2, MIR, Itália ${ }^{\circledR}$ ), seguindo as recomendações da ATS/ERS-2005 ${ }^{19}$, e o parâmetro avaliado foi o percentual do previsto do volume expiratório forçado no primeiro segundo $\left(\mathrm{VEF}_{1}\right)^{20}$. O TC6 foi realizado conforme as recomendações da ATS-2002 ${ }^{21}$, e os parâmetros avaliados foram: distância total percorrida e saturação de oxigênio inicial e final. Foi considerada queda de saturação significativa variação $\geq 4 \%$. A distância percorrida foi apresentada em valores de escore $z$ do previsto $(\text { ZTC6 })^{22}$.

Junto aos pais e/ou responsável, foram obtidas as informações relativas à condição socioeconômica e cultural, quando os pacientes e seus responsáveis foram convidados a participar do estudo. Em caso de aceitação, assinaram o termo de consentimento informado livre e esclarecido. Esta pesquisa obteve aprovação pelos comitês de ética em pesquisa 
dos dois hospitais envolvidos, respeitando a resolução 196/96 do Conselho Nacional de Saúde.

Os dados foram armazenados em um banco de dados elaborado através do programa Statistical Package for the Social Sciences (SPSS), versão 14.0. Os resultados estão expressos da seguinte forma: variáveis quantitativas por média e desvio padrão (DP) quando distribuição simétrica, e mediana e amplitude interquartil quando assimétrica; variáveis categóricas por freqüências absolutas e percentuais. A significância estatística foi de $5 \%(p \leq 0,05)$. O teste $t$ de Student ou análise de variância (ANOVA) foram usados para comparação de variáveis quantitativas com distribuição simétrica. Utilizaram-se o teste qui-quadrado de Pearson ou exato de Fisher para avaliar associações entre as variáveis categóricas. Para avaliar as associações entre as variáveis quantitativas, as correlações de Pearson (distribuição simétrica) ou Spearman (distribuição assimétrica) foram utilizadas. Para avaliar a concordância entre os métodos, realizou-se o teste kappa.

\section{Resultados}

Foram avaliados 57 pacientes, dos quais $40(70,2 \%)$ eram do sexo masculino e $40(70,2 \%)$ da raça branca. A média da idade no momento da avaliação foi de 8,7 anos ( $\pm 4,2$ anos), com variação de 1,5 a 18,9 anos, sendo 37 (64,9\%) crianças abaixo de 10 anos. Em relação ao estágio de maturação sexual dos adolescentes, sete (35\%) foram classificados em prépúbere; oito $(40 \%)$ em púbere e cinco $(25 \%)$ em póspúberes. A mediana de idade em que a criança passou a apresentar as primeiras manifestações clínicas da doença (primeiro episódio de sibilância) foi de 5 meses (P25 = 3,0; P75 = 9,0 meses). A mediana de idade no diagnóstico foi de 12 meses ( $P 25=9,0 ; P 75=17$ meses). A média de peso ao nascer foi de $3.012,7 \mathrm{~g}( \pm 834,5 \mathrm{~g})$, sendo que, destes, 11 pacientes $(22 \%)$ apresentaram peso $<2.500$ g e 11 pacientes $(22 \%)$ eram pré-termo. Em relação à situação socioeconômica familiar, referente à escolaridade da mãe e/ou responsável legal, a média em anos de estudo foi $6,6( \pm 2,9)$ e a mediana da renda familiar per capita, em salários-mínimos, foi de 0,52 $(P 25=0,36 ; P 75=0,74)$.

No que diz respeito ao estado nutricional, encontrou-se 24 pacientes $(42,1 \%)$ com eutrofia, $12(21,1 \%)$ com desnutrição, $10(17,5 \%)$ com risco nutricional para desnutrição, cinco $(8,8 \%)$ com sobrepeso e seis pacientes $(10,5 \%)$ com obesidade. Quando estratificados por faixa etária, os dados referentes ao estado nutricional de crianças e adolescentes são apresentados na Tabela 1.

Os dados referentes à composição corporal de toda a amostra encontram-se descritos na Tabela 2.

Nas análises da associação entre estado nutricional e composição corporal, os pacientes, quanto à categorização do estado nutricional, foram agrupados em três grupos: eutrofia, desnutrição e/ou risco para desnutrição e sobrepeso e/ou obesidade. Observou-se que os pacientes com baixa reserva muscular, por CMB, apresentaram associação significativa com os pacientes desnutridos/risco $(p=0,002)$. Em relação ao excesso de adiposidade, observa-se que, quando se utiliza DCT e DCS, os desnutridos apresentam associação significativa com baixa reserva de gordura. Os pacientes com sobrepeso e obesidade apresentaram associação significativa com alta reserva de gordura com emprego da DCT, DCS e SDCTS. Cabe destacar que, entre os eutróficos, oito (33,3\%) apresentaram baixa reserva muscular e três $(12,5 \%)$ estavam com baixa reserva de gordura ( $p=0,002 ; p=0,001)$.

$\mathrm{Na}$ análise da função pulmonar, de um total de 32 pacientes, três $(9,3 \%)$ não conseguiram executar as manobras necessárias para os testes (espirometria e TC6) e um paciente $(3,1 \%)$ apresentou piora do quadro clínico, impossibilitando a conclusão dos testes no período do estudo. Um paciente apresentou exacerbação dos sintomas, embora houvesse realizado espirometria, e não conseguiu concluir o TC6. Os dados referentes ao estado nutricional, composição corporal, espirometria e TC6 estão descritos na Tabela 3.

Quando avaliada a distância percorrida ZTC6 nos três grupos, desnutridos/risco (média $=-2,99 Z$; DP $=1,43$ ), eutrófi$\cos$ (média $=-1,93 Z ; D P=1,05$ ) e sobrepeso/obesidade (média $=-1,99 Z ; D P=0,81$ ), não houve diferença estatisticamente significativa entre os grupos ( $p=0,105)$. Porém, quando se dividiu os pacientes em dois grupos, sendo o grupo 1 os pacientes desnutridos e/ou risco para desnutrição, e o grupo 2 os pacientes com eutrofia e sobrepeso/obesidade, houve diferença estatisticamente significativa ( $p=0,032)$, sendo que os pacientes com desnutrição e risco nutricional para desnutrição apresentavam ZTC6 significativamente mais baixos que o outro grupo. Analisando-se a associação entre TC6 e reserva muscular, observou-se que os pacientes com baixa reserva muscular apresentavam ZTC6 significativamente mais baixos que os que apresentavam reserva média $(p=0,030)$. A Figura 1 mostra que houve uma diferença média de $1 \mathrm{DP}$ entre os dois grupos, tanto para estado nutricional como para composição corporal.

Houve associação positiva estatisticamente significativa entre o $\operatorname{VEF}_{1} \%$ e o ZTC6 $(r=0,434 ; p=0,024)$, indicando que quanto menor o $\mathrm{VEF}_{1} \%$, menor a distância percorrida. Não se observou associação significativa entre a queda da saturação de oxigênio com o $\operatorname{VEF}_{1} \%$ e com variáveis nutricionais (estado nutricional e composição corporal) $(p>0,05)$. A função pulmonar, avaliada pelo $\operatorname{VEF}_{1} \%$, também não apresentou associação significativa com as variáveis nutricionais $(p>0,05)$.

\section{Discussão}

Os dados encontrados no presente estudo no que se refere à idade de diagnóstico e início das primeiras manifestações clínicas mostraram-se semelhantes a outros estudos, os quais verificaram que a idade de diagnóstico variou entre 1 mês e 3 $\operatorname{anos}^{4,8,23}$, as primeiras manifestações respiratórias ocorreram invariavelmente antes dos $2 \operatorname{anos}^{4}$, e a freqüência maior era observada entre os meninos $7,8,23$. No presente estudo, 
Tabela 1 - Distribuição das crianças e dos adolescentes com bronquiolite obliterante pós-infecciosa de acordo com a categorização do estado nutricional pelo escore $z$ e índice de massa corporal

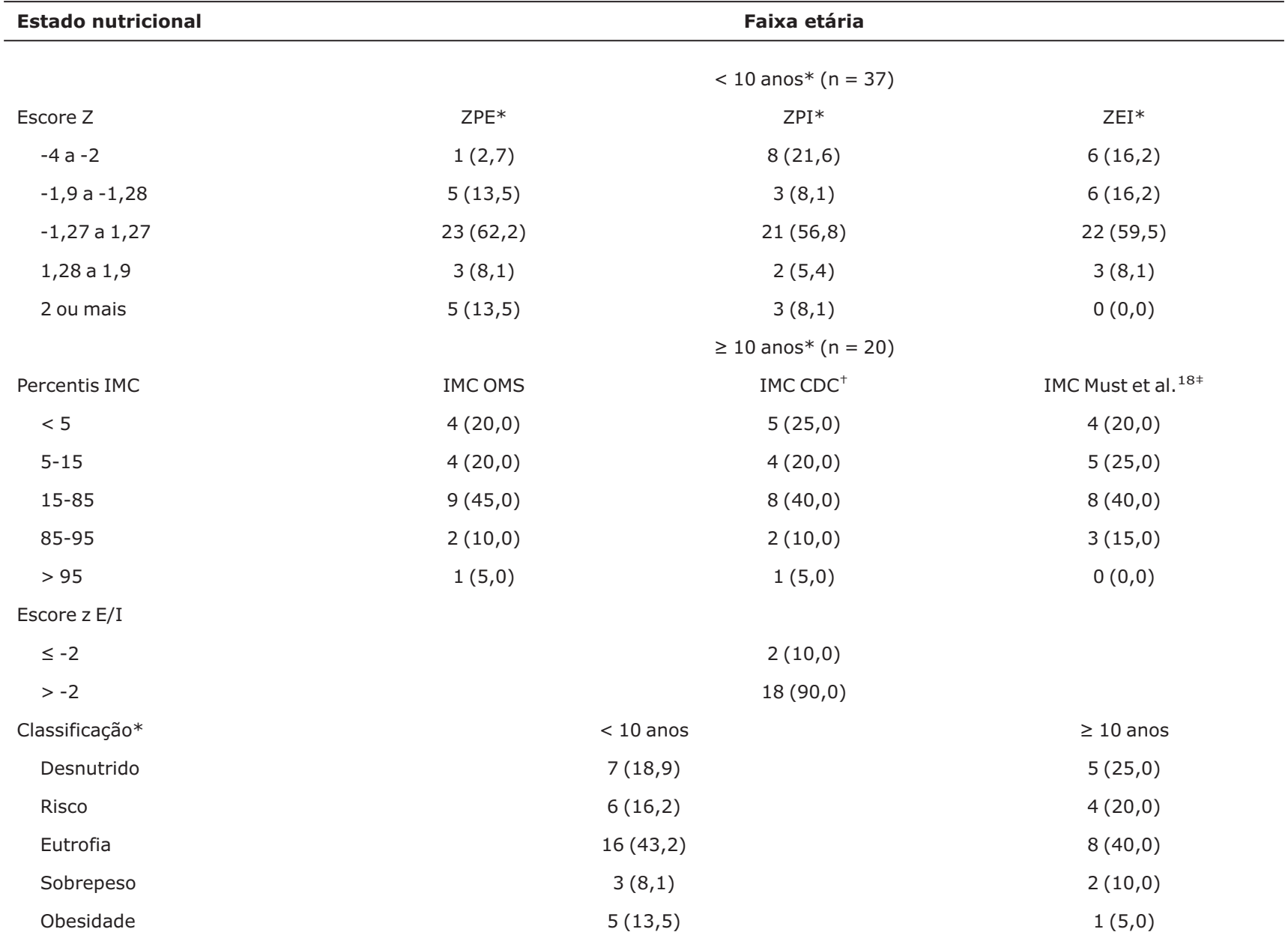

IMC CDC $=$ índice de massa corporal por percentil CDC, (2000); IMC Must et al $.^{18}=$ índice de massa corporal por percentil Must et al. ${ }^{18}$; IMC OMS $=$ Índice de massa corporal por percentil OMS ${ }^{17}$; ZEI = escore $z$ estatura/idade; ZPE = escore $z$ peso/estatura; ZPI = escore z peso/idade.

$* \mathrm{n}(\%)$.

+ Coeficiente kappa de concordância com CDC $(k=0,785), p<0,001$ e com Must et al. ${ }^{18}(k=0,735), p<0,001$

${ }^{\ddagger}$ Coeficiente kappa de concordância com Must et al. ${ }^{18}(\mathrm{k}=0,803), \mathrm{p}<0,001$.

Tabela 2 - Distribuição da amostra de crianças e adolescentes com bronquiolite obliterante pós-infecciosa de acordo com a classificação da composição corporal $(n=57)$

\begin{tabular}{lccc}
\hline Variáveis & & Percentis/categorização \\
\hline Composição corporal & $<5$ & $5-95$ & $>95$ \\
Circunferência braquial & $27(47,4)$ & $28(49,1)$ & $2(3,5)$ \\
Reserva muscular & $<5 /$ baixa & $5-95 /$ média & $>95 /$ alta \\
CMB* & $29(50,9)$ & $27(47,4)$ & $1(1,8)$ \\
Reserva de gordura & $<5 /$ baixa & $5-85 /$ média & $>85 /$ excesso \\
DCT* & $7(12,3)$ & $39(68,4)$ & $11(19,3)$ \\
DCS* & $3(5,3)$ & $37(64,9)$ & $17(29,8)$ \\
SDCTS* & $9(15,8)$ & $36(63,2)$ & $12(21,0)$
\end{tabular}

CMB = circunferência muscular do braço; DCS = dobra cutânea subescapular; DCT = dobra cutânea tricipital; SDCTS = soma das dobras cutâneas tricipital e subescapular.

$* \mathrm{n}(\%)$. 
Tabela 3 - Distribuição das características gerais dos pacientes com bronquiolite obliterante pós-infecciosa que realizaram testes de função pulmonar

\begin{tabular}{|c|c|c|}
\hline Características & $(n=28)$ & IC95\% \\
\hline \multicolumn{3}{|l|}{ Estado nutricional* } \\
\hline Desnutrição e/ou risco & $12(42,9)$ & $24,6-61,2$ \\
\hline Eutrofia & $12(42,9)$ & $24,6-61,2$ \\
\hline Sobrepeso/obesidade & $4(14,3)$ & $1,3-27,3$ \\
\hline \multicolumn{3}{|l|}{ Composição corporal } \\
\hline \multicolumn{3}{|l|}{ Reserva muscular (CMB)* } \\
\hline Baixa & $17(60,7)$ & $42,6-78,8$ \\
\hline Média & $11(39,3)$ & $21,2-57,4$ \\
\hline Alta & $0(0,0)$ & - \\
\hline \multicolumn{3}{|c|}{ Reserva de gordura (SDCTS)* } \\
\hline Baixa & $2(7,1)$ & $0,0-16,6$ \\
\hline Média & $20(71,4)$ & $54,7-88,1$ \\
\hline Alta & $6(21,4)$ & $6,2-36,6$ \\
\hline \multicolumn{3}{|l|}{$\operatorname{VEF}_{1}$ Classificação* } \\
\hline DVO leve & $5(17,8)$ & $3,6-32,0$ \\
\hline DVO moderado & $15(53,5)$ & $35,0-72,0$ \\
\hline DVO grave & $8(28,5)$ & $11,8-45,2$ \\
\hline \multicolumn{3}{|l|}{$\mathrm{TC6}^{+}(\mathrm{n}=27)$} \\
\hline Escore $z^{\ddagger}$ & $-2,37 \pm 1,2$ & $-2,87$ a $-1,87$ \\
\hline Distância percorrida* & $507,7 \pm 70,8$ & $479,7-535,8$ \\
\hline \multicolumn{3}{|l|}{ Saturação de oxigênio } \\
\hline Saturação inicial ${ }^{\ddagger}$ & $97,3 \pm 1,07$ & $96,9-97,7$ \\
\hline Saturação final ${ }^{\ddagger}$ & $95,3 \pm 3,3$ & $94,0-96,7$ \\
\hline Queda na saturação* & $12(44,4)$ & $26,0-62,8$ \\
\hline
\end{tabular}

CMB = circunferência muscular do braço; DVO = distúrbio ventilatório obstrutivo; IC95\% = intervalo de $95 \%$ de confiança; SDCTS = soma das dobras

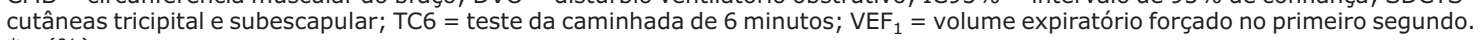

* $\mathrm{n}(\%)$.

+ Não concluiu o teste $=1(3,5 \%)$.

* Média \pm desvio padrão

houve um predomínio de crianças da raça branca, e parece estar relacionado às características da população de onde a amostra foi coletada.

O papel dos fatores socioeconômicos sobre a morbimortalidade das doenças respiratórias na infância é bem descrito na literatura ${ }^{24}$, em que a situação socioeconômica e demográfica desfavorável interfere de maneira imperativa no estabelecimento e no curso das doenças infantis ${ }^{25}$. Cabe destacar que se encontrou um alto percentual de famílias de baixa renda familiar per capita e baixa escolaridade materna, que pode ser explicado pelo fato de a amostra ser proveniente de dois hospitais públicos de atendimento terciário em um país em desenvolvimento. Caldwell ${ }^{26}$ apresentou evidências do efeito positivo e independente da escolaridade materna sobre as taxas de mortalidade infantil e já reconhecia a importância do ambiente como fator interveniente dessa associação.
A literatura é escassa em relação à avaliação do estado nutricional de pacientes acometidos por BO. Não existe referencial científico publicado que se assemelhe com a metodologia utilizada neste trabalho. Por este motivo, podemos caracterizar este estudo como inédito no que se refere à avaliação do estado nutricional e composição corporal em crianças e adolescentes com esta enfermidade.

Os indivíduos avaliados neste estudo são acompanhados por equipe multidisciplinar, na qual a preocupação com o estado nutricional é uma constante. Chama atenção o fato de ter-se encontrado uma alta prevalência de desnutrição $(21,1 \%)$ e risco para desnutrição $(17,5 \%)$. Esta situação provavelmente deve-se, além da morbidade decorrente da BO, como infecções recorrentes, episódios de exacerbação e hospitalização freqüentes, à aplicação de critérios mais sensíveis 


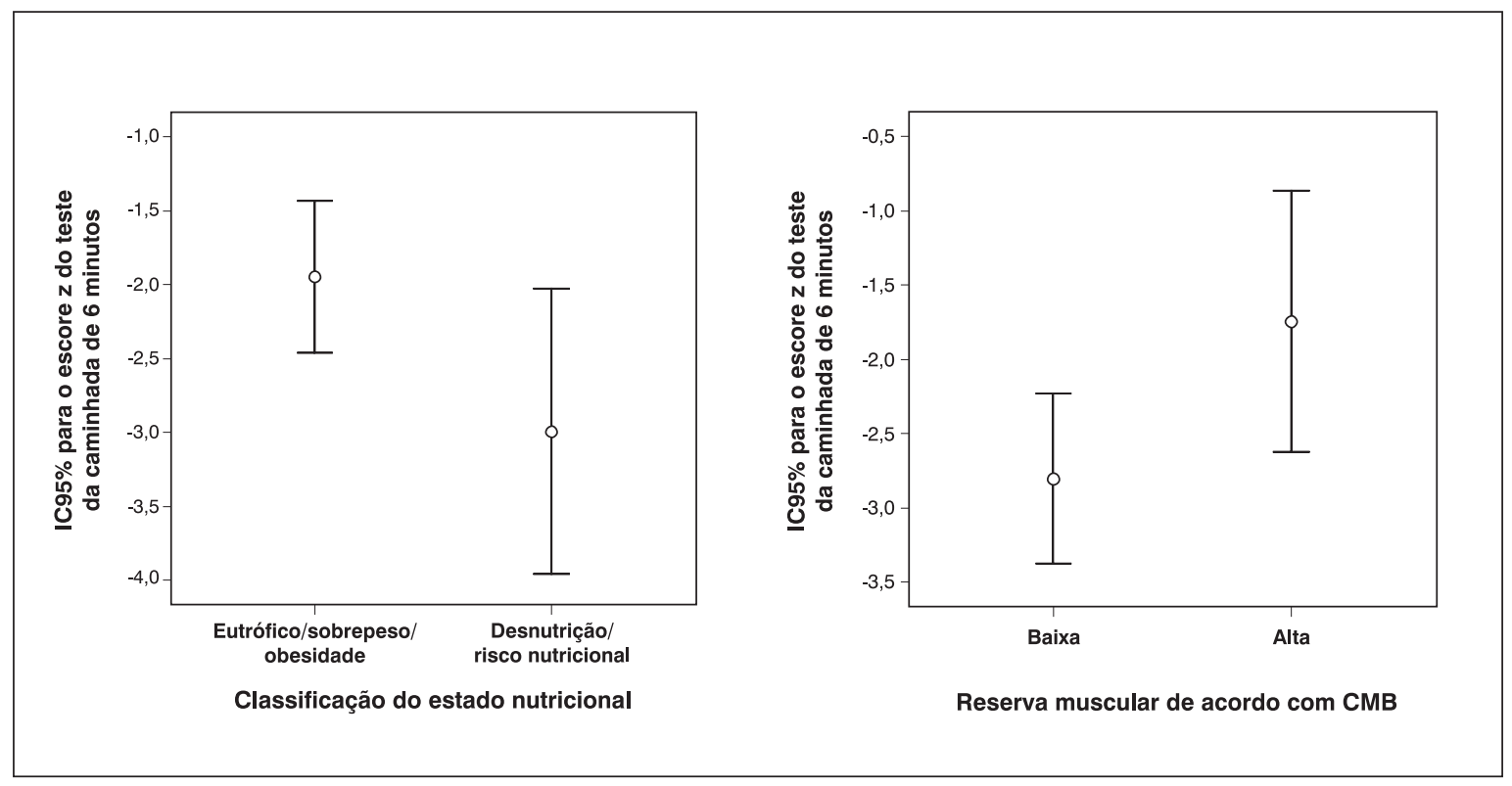

* CMB $=$ circunferência muscular do braço.

Figura 1 - Distribuição da avaliação do escore z do teste da caminhada de 6 minutos de acordo com a classificação do estado nutricional e reserva muscular, pela circunferência muscular do braço dos pacientes maiores de 8 anos com bronquiolite obliterante pós-infecciosa

de avaliação do estado nutricional, analisando diversos indicadores para a caracterização ou classificação do estado nutricional, pois sabe-se que nenhum destes apresenta um caráter universal. Mesmo sem detalhar os métodos de diagnóstico nutricional, ou limitando-se à aplicação de um único índice, a desnutrição, também mostrou-se um achado freqüente nos poucos estudos realizados em $\mathrm{BO}^{4,5,7,8}$.

Mocelin et al. ${ }^{7}$ observaram que, de 19 pacientes entre $7 \mathrm{e}$ 15 anos, utilizando o ZPE, cinco $(26,31 \%)$ apresentavam desnutrição leve $[-2 \leq z<-1]$, um paciente $(5,2 \%)$ apresentava desnutrição moderada ( $<-2 \mathrm{z}$ ) e os demais eram eutróficos, dados bem inferiores aos achados do presente estudo, que encontrou $21,1 \%$ de desnutrição e $17,5 \%$ em risco para desnutrição. Os autores, para avaliar o estado nutricional dos pacientes, utilizaram somente o índice $\mathrm{PE}$, que reflete um comprometimento mais recente do crescimento com reflexo mais pronunciado no peso, não levando em consideração a idade do indivíduo ${ }^{10,27}$.

Não há relatos na literatura referentes a pacientes com BO que apresentam excesso de peso. Neste estudo, observou-se que $8,8 \%$ dos pacientes apresentavam sobrepeso e $10,5 \%$ obesidade, chegando-se a valores aproximados daqueles obtidos em estudo realizado na população saudável ${ }^{28}$.

Entre as crianças, em relação aos índices aplicados, observou-se que o PI e o EI apresentaram maior percentual de desnutrição, 21,6 e 16,2\%, respectivamente, ao passo que o PE subestimou o diagnóstico de desnutrição. Entre os adolescentes, a aplicação do IMC demonstrou um alto percentual de pacientes com desnutrição ( $25 \%$ ) e risco para desnutrição (20\%). Estes resultados devem ser analisados levando-se em consideração as particularidades existentes entre os diferentes índices utilizados. O uso do PI é muito adequado para o acompanhamento do crescimento ponderal infantil e reflete a situação global do indivíduo, porém não diferencia o comprometimento nutricional agudo dos crônicos. Já o EI expressa o crescimento linear. O comprometimento do índice EI sugere que o crescimento da criança é afetado a longo prazo 10,27,29.

Em relação à composição corporal, foi encontrada depleção de reserva muscular estimada pela CMB em 50,9\% dos pacientes estudados, e depleção de reserva de gordura por DCT, DCS e SDCTS ocorreu em 12,3, 5,3 e 15,8\%, respectivamente. Fiates et al. ${ }^{30}$, analisando a composição corporal de pacientes com FC, encontrou por avaliação da CMB que $46,1 \%$ dos pacientes apresentavam depleção de massa muscular e $30,7 \%$ de depleção de reserva de gordura por DCT. A inexistência de estudos em $B O$ no que se refere à composição corporal fez com que as comparações fossem realizadas entre diferentes doenças. Na FC, além do comprometimento pulmonar, muitos dos pacientes apresentam manifestações digestórias envolvidas. Portanto, em relação a comparações com a BO, essas devem ser realizadas levando-se em consideração tais particularidades.

No grupo classificado como eutrófico, destaca-se o fato de que $33,3 \%$ deles apresentaram baixa reserva muscular e $12 \%$, baixa reserva de gordura. Estes resultados salientam a necessidade de uma avaliação do estado nutricional mais completa, especialmente entre pacientes com doença crônica. No presente estudo, se não fosse realizada uma avaliação mais abrangente, associando-se indicadores de peso e 
estatura a dados de composição corporal, até 45,3\% dos pacientes eutróficos, os quais já apresentavam alguma forma de desnutrição (depleção de reserva muscular e/ou baixa reserva de adiposidade), não seriam identificados e adequadamente manejados.

Dentre os pacientes $(n=28)$ que realizaram testes de função pulmonar, salienta-se o alto percentual dos que apresentaram desnutrição e/ou risco para desnutrição (42,9\%) e baixa reserva muscular $(60,7 \%)$. Estes dados indicam a comprometimento do estado nutricional destes indivíduos.

Na amostra estudada, encontrou-se que o prejuízo da função pulmonar se associou com menor desempenho no TC6. Este fato pode estar relacionado com a condição dos pacientes, os quais, na sua maioria, apresentavam distúrbio ventilatório obstrutivo de moderado a grave. O diagnóstico de desnutrição ou risco nutricional, bem como a baixa reserva muscular, apresentaram associação significativa com TC6, indicando o grau de limitação funcional destes pacientes. Nesta amostra, pode-se inferir que a deterioração do estado nutricional tem relação direta no desempenho ao exercício.

As associações entre os achados da espirometria $\left(\operatorname{VEF}_{1} \%\right)$ e variáveis nutricionais não foram significativas, devendo-se considerar que, por tratar-se de uma doença rara, uma pequena amostra de pacientes realizou espirometria. Essa população apresentou um importante déficit nutricional, além de ter demonstrado ser relativamente homogênea em relação ao grau de comprometimento pulmonar, em que $82 \%$ dos pacientes apresentaram distúrbio ventilatório obstrutivo moderado ou grave.

Estes achados podem refletir a morbidade associada à $\mathrm{BO}$ e reforçam a necessidade do acompanhamento multidisciplinar nestes pacientes, a exemplo de outras pneumopatias crônicas, como a FC. Destaca-se a importância da assistência nutricional sistemática, bem como a realização periódica e detalhada da avaliação do estado nutricional desses pacientes. A nutrição pode vir a ser um importante fator prognóstico na evolução da BO, visto a importância do crescimento pulmonar acompanhar o crescimento somático.

Nesta pesquisa, os pacientes apresentaram importante comprometimento nutricional, apontando que tanto o prejuízo funcional pulmonar quanto a desnutrição estão associados com pior desempenho ao exercício no TC6. Estudos futuros são necessários na tentativa de elucidar se a desnutrição e a baixa reserva muscular são conseqüências inerentes da enfermidade ou se esta condição poderá ser revertida com a aplicação de uma terapia nutricional mais específica.

\section{Agradecimentos}

Agradecemos ao CNPq, pelo apoio financeiro, a Vânia Naomi Hirakata (Grupo de Pesquisa e Pós-Graduação do Hospital de Clínicas de Porto Alegre - HCPA) e a Ceres Oliveira, pelas suas contribuições com as análises estatísticas.

\section{Referências}

1. Hardy KA. Childhood bronchiolitis obliterans. In: Epler G, editor. Diseases of the bronchioles. New York: Raven Press; 1994.

2. Teper A, Fischer GB, Jones MH. Seqüelas respiratórias de doenças virais: do diagnóstico ao tratamento. J Pediatr (Rio J). 2002;78 Suppl 2:S187-94.

3. Hardy KA, Schidlow DV, Zaeri N. Obliterative bronchiolitis in children. Chest. 1988;93:460-6.

4. Lobo AL, Guardiano M, Nunes T, Azevedo I, Vaz LG. Bronquiolite obliterante pós-infecciosa na criança. Rev Port Pneumol. 2007; 13:495-509.

5. Teper AM, Kofman CD, Maffey AF, Vidaurreta SM. Lung function in infants with chronic pulmonary disease after severe adenoviral illness. J Pediatr. 1999;134:730-3.

6. Chan PW, Muridan R, Debruyne JA. Bronchiolitis obliterans in children: clinical profile and diagnosis. Respirology. 2000; 5:369-75.

7. Mocelin H, Fischer G, Iriar K. Evaluación clínica y funcional de niños con bronquiolitis obliterante post-infecciosa con seguimento a largo prazo. Rev Chil Pediatr. 2004;75:12-7.

8. Colom AJ, Teper AM, Vollmer WM, Diette GB. Risk factors for the development of bronchiolitis obliterans in children with bronchiolitis. Thorax. 2006;61:503-6.

9. Milner AD, Murray M. Acute bronchiolitis in infancy: treatment and prognosis. Thorax. 1989;44:1-5.

10. World Health Organization (WHO). Pysical status: the use and interpretation of antropometry. Geneva: WHO; 1995.

11. Frisancho AR. Anthropometric standards for the assessment of growth and nutritional status. Ann Arbor: University of Michigan Press; 1990.

12. Teitelbaum D, Guenter P, Howell WH, Kochevar ME, Roth J, Seidner DL. Definition of terms, style, and conventions used in A. S. P. E. N. guidelines and standards. Nutr Clin Pract. 2005; 20:281-5

13. Nogueira-De-Almeida CA, Ricco RG, Nogueira MP, Del Ciampo LA, Muccillo G. Avaliação do uso do percentil 10 de peso para idade como ponto de corte para deteç̧ão de crianças sob risco nutricional. J Pediatr (Rio J). 1999;75:345-9.

14. Tanner J. Growth at adolescence. 2nd ed. Oxford: Blackwell Scientific; 1962.

15. WHO Multicentre Growth Reference Study Group. WHO Child Growth Standards based on length/height, weight and age. Acta Paediatr Suppl. 2006;450:76-85.

16. National Center for Disease Control do National Center for Health Statistics. 2000 CDC Growth Charts: United States. http:// www.cdc.gov/growthcharts/. Acesso: 20/02/2008.

17. de Onis M, Onyango AW, Borghi E, Siyam A, Nishida C, Siekmann J. Development of a WHO growth reference for school-aged children and adolescents. Bull World Health Organ. 2007; 85:660-7.

18. Must A, Dallal GE, Dietz WH. Reference data for obesity: 85th and 95th percentiles of body mass index (wt/ht2) and triceps skinfold thickness. Am J Clin Nutr. 1991;53:839-46.

19. Miller MR, Hankinson J, Brusasco V, Burgos F, Casaburi R, Coates A, et al; ATS/ERS Task Force. Standardisation of spirometry. Eur Respir J. 2005;26:319-38.

20. Knudson RJ, Lebowitz MD, Holberg CJ, Burrows B. Changes in the normal maximal expiratory flow-volume curve with growth and aging. Am Rev Respir Dis. 1983;127:725-34. 
21. ATS Committee on Proficiency Standards for Clinical Pulmonary Function Laboratories. ATS statement: guidelines for the six-minute walk test. Am J Respir Crit Care Med. 2002; 166:111-7.

22. Geiger R, Strasak A, Treml B, Gasser K, Kleinsasser A, Fischer V, et al. Six-minute walk test in children and adolescents. J Pediatr. 2007;150:395-9.

23. dos Santos RV, Rosário NA, Ried CA. Bronquiolite obliterante pós-infecciosa: aspectos clínicos e exames complementares de 48 crianças. J Bras Pneumol. 2004;30:20-5.

24. Prietsch SO, Fischer GB, Cesar JA, Fabris AR, Mehanna $H$, Ferreira $\mathrm{TH}$, et al. Doença aguda das vias aéreas inferiores em menores de cinco anos: influência do ambiente doméstico e do tabagismo materno. J Pediatr (Rio J). 2002;78:415-22.

25. Carvalhaes MA, Benício MH. Capacidade materna de cuidar e desnutrição infantil. Rev Saude Publica. 2002;36:188-97.

26. Caldwell JC. Education as a factor in mortality decline: an examination of Nigeria data. Population Studies. 1979; 33:395-413.
27. Brasil. Ministério da Saúde. Vigilância alimentar e nutricional SISVAN: orientações básicas para a coleta, processamento, análise de dados e informações em serviços de saúde. Brasília: Ministério da Saúde; 2004.

28. Instituto Brasileiro de Geografia e Estatística. Pesquisa de Orçamentos Familiares (POF) 2002-2003: análise da disponibilidade domiciliar de alimentos e do estado nutricional no Brasil. Rio de Janeiro: IBGE; 2004.

29. Waterlow J. Evaluación del estado nutricional en la comunidad. In: Waterlow JC, editor. Malnutrición proteico-energética. Washington, DC: OPS; 1996.

30. Fiates GM, Barbosa E, Auler F, Feiten SF, Miranda F. Estado nutricional e ingestão alimentar de pessoas com fibrose cística. Rev Nutr. 2001;14:95-101.

Correspondência:

Vera Lúcia Bosa

Rua General Lima e Silva, 707/801

CEP 90050-101 - Porto Alegre, RS

Tel.: (51) 3212.2951, (51) 3214.8646

Fax: (51) 3214.8646

E-mail: vlbosa@terra.com.br 